\title{
THE BLOOD SUPPLY OF THE TALUS
}

\author{
G. L. Mulfinger, Pasadena, United States of America \\ and J. Trueta, Barcelona, Spain \\ From the Nuffield Orthopaedic Centre, Oxford, England
}

It has long been known that avascular necrosis of the talus may occur as a sequel to certain fractures and dislocations. Consequently the blood supply to the talus has been the subject of considerable investigation. The arterial supply to the talus has been well described in most of its aspects but the arterial distribution and the anastomoses within the bone have not been elucidated completely despite their significant surgical implications. The present study was undertaken because modern techniques are particularly applicable in showing the extraosseous and intraosseous arterial supply.

The first attempt to inject the vessels of the talus with a radiopaque substance and to study their distribution radiologically was by Lexer, Kuliga and Türk (1904). They said that there were many vessels in the talus but made no mention of their origin or of their pattern within the talus. Sneed (1925) from arterial injection in five specimens, concluded that there was no main nutrient artery but rather numerous small blood vessels. Similar opinions were expressed by Watson-Jones (1940) and Kleiger (1948). Phemister (1940) reported that the major vessel to the talus was the dorsalis pedis artery and that its branches were distributed mainly to the junction of the neck and body of the talus in the tarsal sinus area. McKeever (1943) injected seven legs in order to study the vasculature of the talus. He stated that its entire blood supply appeared to come from a branch of the dorsalis pedis artery which reached the bone through the talo-navicular ligament.

Wildenauer (1950) gave a complete description of the blood supply to the talus and the first full description of the artery which arises from the posterior tibial artery, runs through the tarsal canal and terminates by anastomosing with the artery of the tarsal sinus; he called this vessel the artery of the tarsal canal. He also pointed out the anatomical distinction between the tarsal sinus and the tarsal canal. The tarsal canal is formed by the sulcus of the talus and the sulcus of the calcaneus. It lies obliquely from a postero-medial to an antero-lateral position and opens into the tarsal sinus. The canal contains the interosseous talo-calcaneal ligament and the artery of the tarsal canal. Further, Wildenauer believed that the most important vascular contributions came from the arteries of the tarsal sinus, tarsal canal and medial periosteal network.

Coltart (1952) listed three areas as the most important in relation to the arterial supply: the upper surface of the neck, the tarsal sinus area and the medial surface of the body. This conclusion was apparently based on clinical observation. He believed that the blood supply to any one of these areas could be destroyed without endangering the viability of the bone.

Lauro and Purpura (1956) found a rich blood supply to the talus by radiological studies after arterial injection of a radiopaque medium, but they did not find a definite pattern of distribution of arteries within the bone. They noted arteries supplying the talus from the sinus tarsi area, a well defined artery that they termed a medial articular branch, and arteries in the talo-calcaneal ligament.

Fourteen amputated legs were injected by Haliburton, Sullivan, Kelly and Peterson (1958) for study of the arterial supply and the intraosseous vascular pattern of the talus. The intraosseous pattern of the head of the talus showed approximately equal contributions from both the superior and the inferior lateral surfaces of the neck. The body received the largest branches through the antero-inferior aspect of the neck. The medial vessels were next in importance, and the lateral and posterior vessels supplied the smallest areas. It is difficult, from their illustrative photographs, to determine which areas are supplied by the various vessels, and no 
distinction was made between arteries and veins. Further studies based on the injection of twenty-five specimens were reported by Montis and Ridola (1959); they attributed prime importance to vessels entering in the region of the sinus tarsi, but they did not delineate the segmental areas supplied by these vessels. Many anastomoses between small vessels were found connecting the upper, lateral and medial areas within the bone. Depreux and Hollingshausen (1963) examined fifty injected specimens. They agreed with the work of Wildenauer (1950) and Coltart (1952) but stressed the separate importance of the supply through the periosteal network and from the major branches from the tarsal canal.

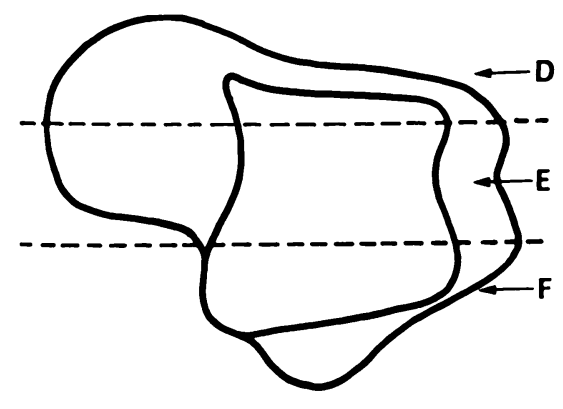
DORSAL VIEW OF THE TALUS
SHOWING THE AREAS COVERED SHOWING THE AREAS COVERED
BY THE FOLOWING SECTIONS

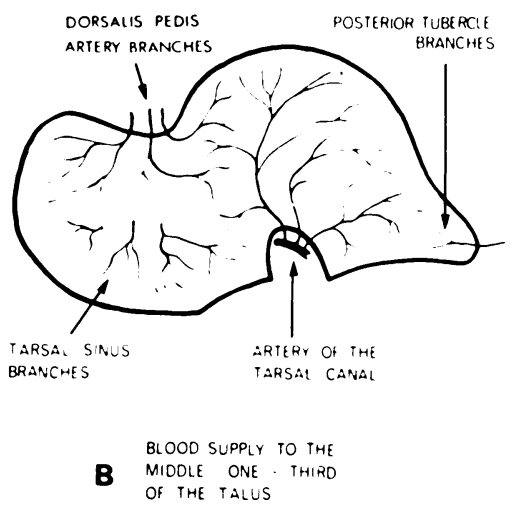

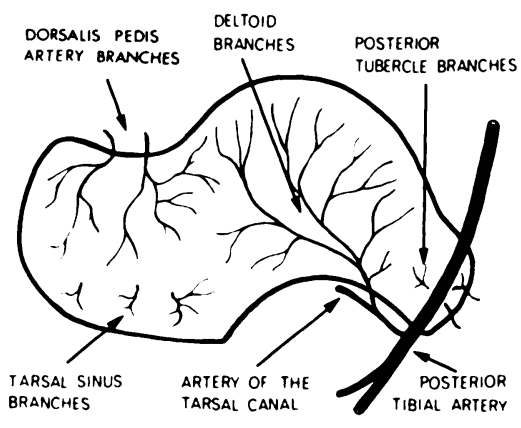

A BLOD SUPPLY TO THE
MEDIAL ONE. THIRD of THE tALUS

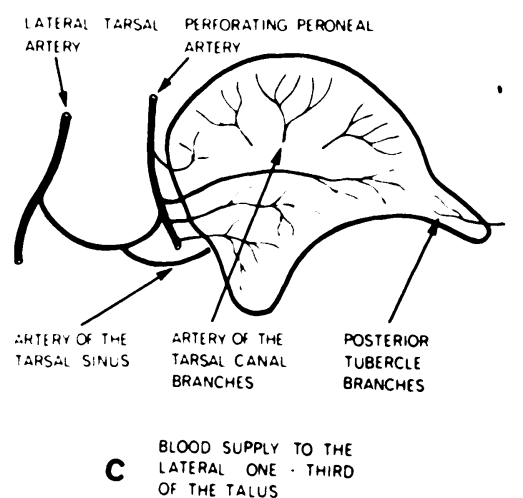

Fici. 1

Diagram to show the blood supply to the talus in sagittal sections.

\section{MATERIALS AND METHODS}

Necropsy specimens free of known arterial disease in the age range of nineteen to seventytwo years were used. The method employed was that of Trueta and Harrison (1953), with injection of a 50 per cent micropaque solution. The arterial perfusions were done between thirty-six and forty-eight hours after death. This time interval allows deterioration of the capillary bed so that the injected material does not usually pass into the venous circulation. There is a definite advantage in studying arterial patterns alone, because arteries and veins in bone cannot always be easily distinguished when they are completely injected.

The injection of the foot was done by cannulation of the femoral artery in half the specimens and by cannulation of the posterior tibial, anterior tibial, and common peroneal arteries in the other half. Injection by the two different methods made no apparent difference in the

VOI. 52 B, NO. I, HFBRUARY 1970

$\mathrm{L}$ 
results. No attempt was made to irrigate the vessels before the injection of Micropaque. After injection the feet were frozen to -4 degrees Centigrade. The whole foot was then sectioned with a saw. Fifteen feet were cut sagittally, eleven coronally, and four transversely into slices which varied in thickness between 5 and 25 millimetres. Fixation was done with 10 per cent neutral formalin and decalcification with 10 per cent nitric acid. The Spalteholz technique was used on all the slices. The vessels were then studied by the naked eye, by dissecting

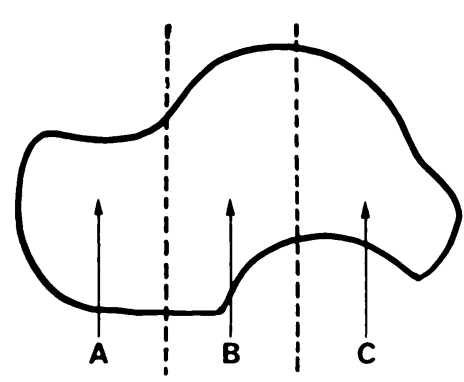

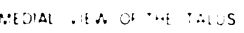
SHOWING ThE DERAS CO.PPE

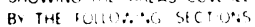

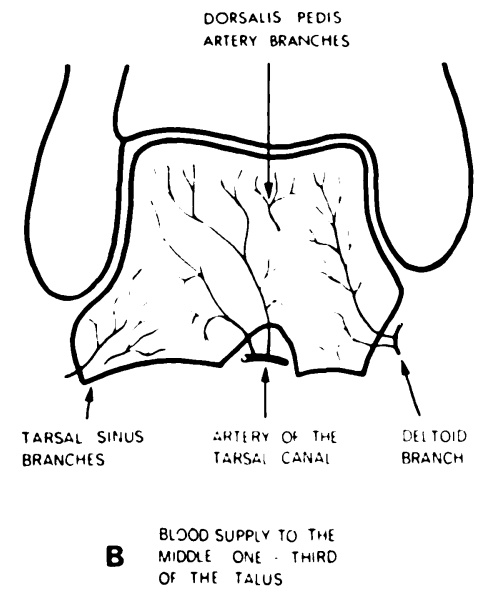

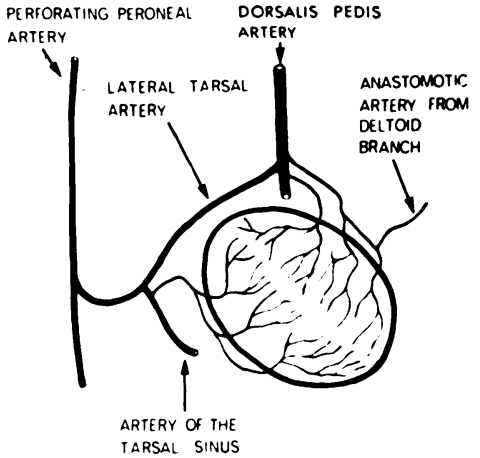
A BLOOD SUPPLY TO THE
HEAD OF THE TALUS

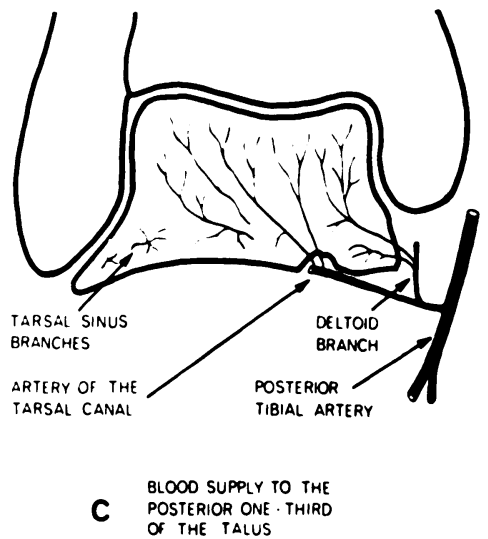

Fig. 2

Diagram to show the blood supply to the talus in coronal sections.

microscope and by fine-grain radiography. With this technique, satisfactory demonstration of the various patterns in the talus was obtained in thirty out of thirty-six specimens, and the results presented in this paper are based on the observations made on the thirty specimens.

\section{EXTRAOSSEOUS ARTERIES}

Posterior tibial artery branches-The first arteries to the talus from the posterior tibial artery are from the calcaneal branches. These small arteries form a network over the posterior tubercle with branches from the peroneal artery.

The artery of the tarsal canal usually arises from the posterior tibial artery about a centimetre proximal to the origin of the medial and lateral plantar arteries (A in Fig. 1). It passes anteriorly between the sheaths of the flexor digitorum longus and flexor hallucis longus tendons to enter the tarsal canal. The artery lies in the dorsal half of the canal, much closer 
to the talus than to the calcaneus (B in Fig. 1 and Fig. 3). Branches into the body are given off in the canal and the largest of these enters about the middle of the body (Fig. 6). A few small branches pass into the calcaneus. The artery of the tarsal canal anastomoses with the artery of the tarsal sinus in the tarsal canal.

About 5 millimetres from the origin of the artery of the tarsal canal a branch is given off to the medial surface of the body of the talus (A in Fig. 1, C in Fig. 2 and Fig. 4). This vessel runs forward between the talo-tibial and talo-calcaneal parts of the deltoid ligament. The artery supplies the medial periosteal surface of the body and anastomoses with dorsalis pedis or anterior tibial artery branches over the neck of the talus. In the present paper this vessel is referred to as the deltoid branch.

Variations-In five specimens the artery of the tarsal canal originated from the medial plantar branch of the posterior tibial artery. In one specimen the vessel was duplicated. In another, no artery was found in this area and the artery of the tarsal sinus was much larger than usual. Anastomosis between the artery of the tarsal canal and the artery of the tarsal sinus was found in all specimens in which both were present, but the size of the anastomotic vessels varied greatly. In four instances the anastomosis was within the bone. The deltoid branch originated from the posterior tibial artery in nine specimens, in two of which it was duplicated.

Anterior tibial artery-The anterior tibial artery or dorsalis pedis artery sends branches to the superior surface of the neck of the talus either as direct medial tarsal artery branches or anterior medial malleolar artery branches. The anterior tibial artery becomes the dorsalis pedis artery at the level of the ankle joint, and the branches to the talus can arise at the joint line or just above it. It is often difficult to determine whether a particular vessel is from the anterior tibial or dorsalis pedis artery.

The sinus tarsi area is supplied mainly by the dorsalis pedis artery with a small contribution from the anterior lateral malleolar branch of the anterior tibial artery and from the perforating peroneal artery. The artery of the tarsal sinus was found in all specimens. It is usually slightly larger than the artery of the tarsal canal. The artery of the tarsal sinus usually starts from an anastomotic loop between the lateral tarsal branch of the dorsalis pedis artery and the perforating peroneal artery (A in Fig. 2). The artery of the tarsal sinus usually gives off a few branches to the head of the talus and then enters the tarsal canal in which, after giving off branches to the body, it anastomoses with the artery of the tarsal canal.

The lateral tarsal artery may also give direct branches to the head of the talus and to the lateral anterior aspect of the body of the talus.

There is an anastomotic network of small arteries in the tarsal sinus. This is formed from lateral tarsal, perforating peroneal, and anterior lateral malleolar artery branches.

Variations-In one specimen the dorsalis pedis artery was absent and the perforating peroneal artery supplied this area. In two specimens the anterior tibial artery bifurcated into medial and lateral divisions proximal to the ankle. The origin of the artery of the tarsal sinus was extremely variable and was seen to arise from any of the anastomotic plexus of arteries situated in the tarsal sinus. In six specimens the artery of the tarsal sinus originated as a direct branch from the dorsalis pedis artery. Because of the great number and complexity of the variations, no attempt is made here to classify them. These have been well summarised by Adachi (1928) and Huber (1941).

Peroneal artery-Small branches from the peroneal artery join with the calcaneal branches of the posterior tibial artery to form a plexus over the posterior tubercle area of the talus. The perforating peroneal artery, as previously mentioned, contributes to the plexus in the tarsal sinus. The artery of the tarsal sinus appeared as a direct branch from the perforating peroneal artery in four specimens.

Variations-In three specimens the perforating peroneal artery could not be identified. The size of the vessel was not constant, and frequently it was very small and did not represent a significant blood supply.

VO'. 52 B, NO. 1, FRBRARY 1970 


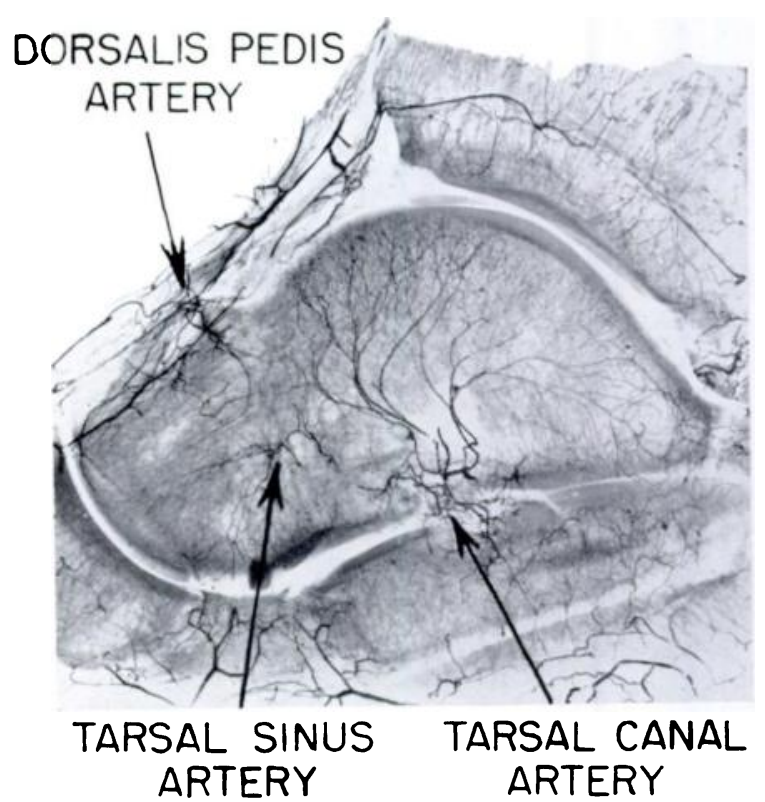

FIG. 3

A sagittal section through the middle third of the talus.
INTRAOSSEOUS ARTERIAL PATTERN

Head-The head of the talus is supplied from two sources: branches from the anterior tibial artery or the dorsalis pedis artery supply the medial superior half, and the lateral and inferior half of the head is supplied directly from the artery of the tarsal sinus, from tranches of the sinus tarsi anastomosis, or from direct branches of the lateral tarsal artery (A in Fig. 2 and Fig. 5).

Variations-The vascular pattern in the head was relatively constant, and no significant variations were seen.

Body-The body of the talus is mostly supplied by the anastomotic artery in the tarsal canal. This artery usually gives four or five main branches into the body which form arcs curving postero-laterally (B in Fig. 1, Figs. 3 and 6). The vessel supplies almost all of the middle third, except for a small area from superior neck arteries. It supplies all of the lateral third except the lateral aspect of the posterior facet and variable amount of the lateral edge of the trochlear part of the body.

Second in importance are the deltoid branches, which enter the body on its medial periosteal surface and supply the medial quarter or third of the body (A in Fig. 1, B and C in Fig. 2 and Fig. 6).

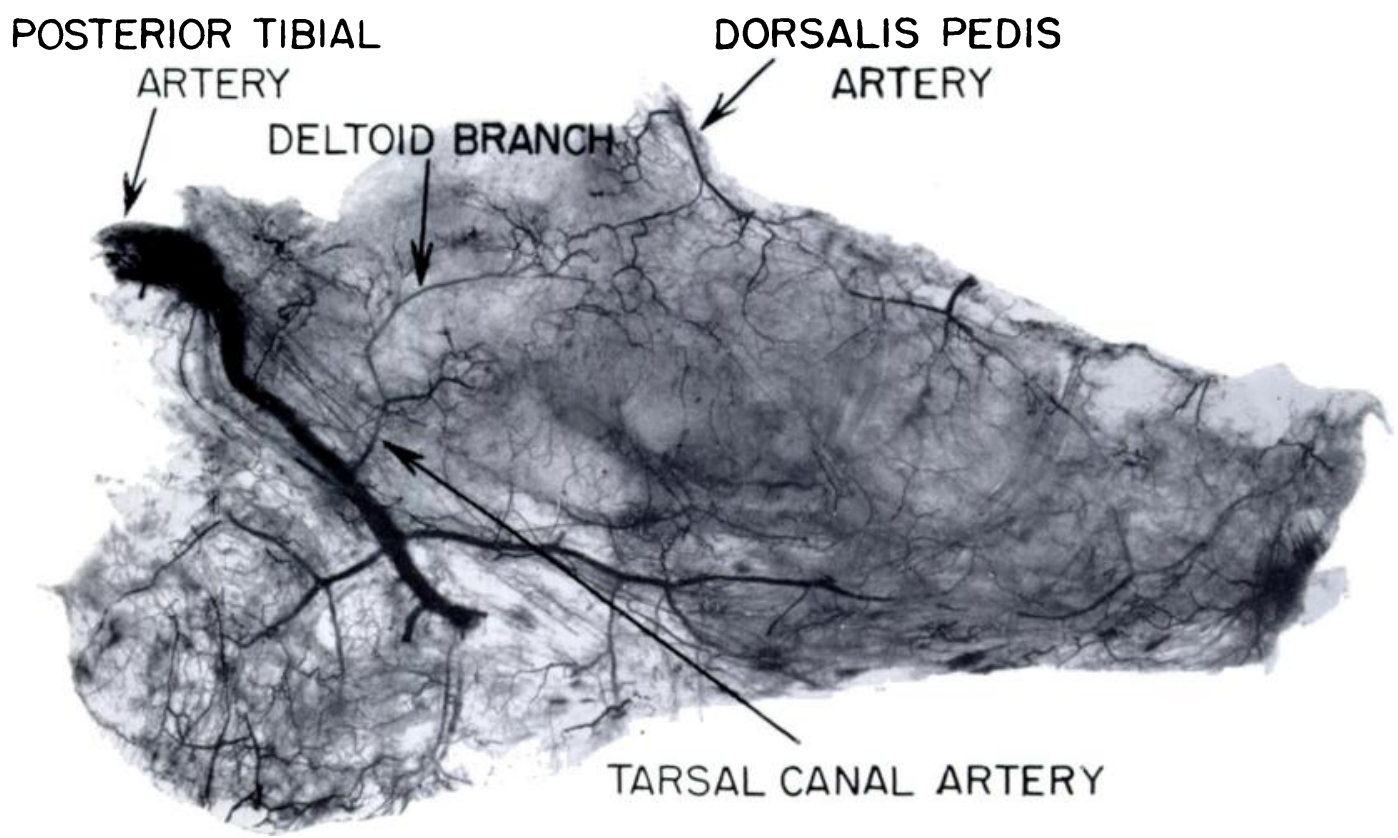

Fig. 4

A sagittal section of the middle third of the tarsal bones. Anastomoses between the deltoid branches and the dorsalis pedis artery branches can be seen.

THE JOURNAL OF BONE AND JOINT SURGERY 
The arteries from the anastomotic network in the tarsal sinus which enter the lateral anterior surface of the body supply its lateral inferior aspect including most of the posterior facet (C in Fig. 1, B in Fig. 2).

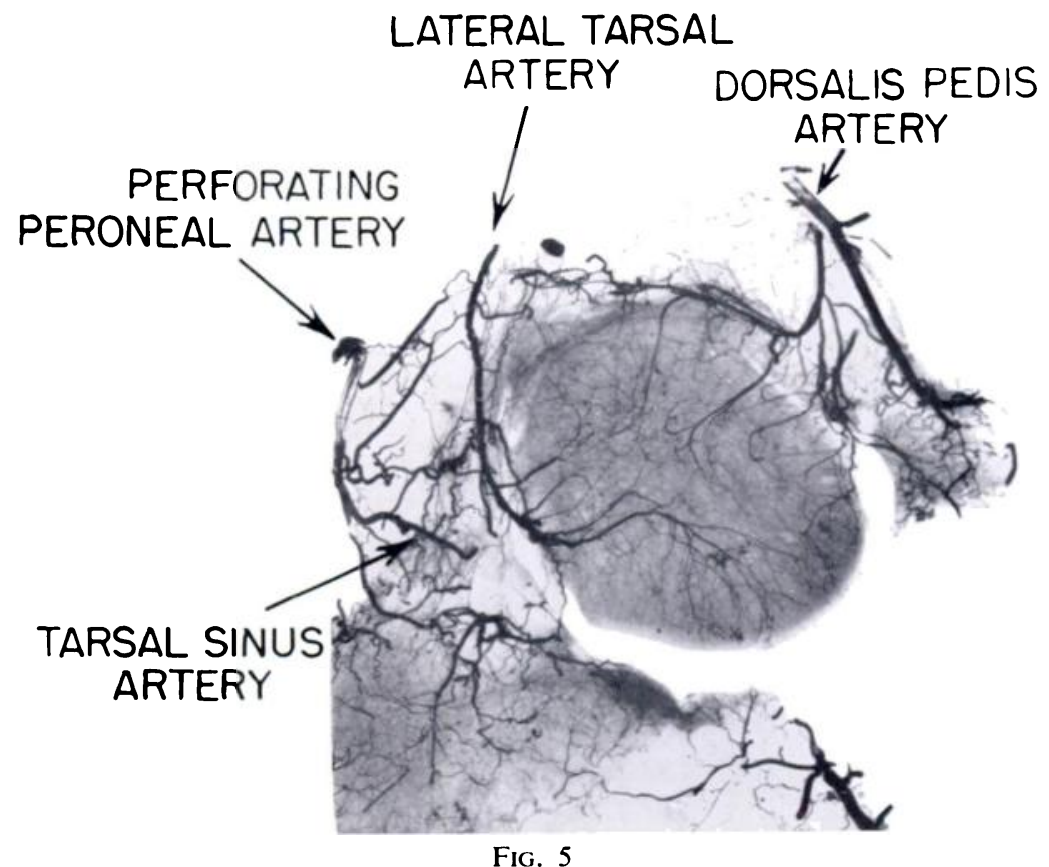

A coronal section through the head of the talus.

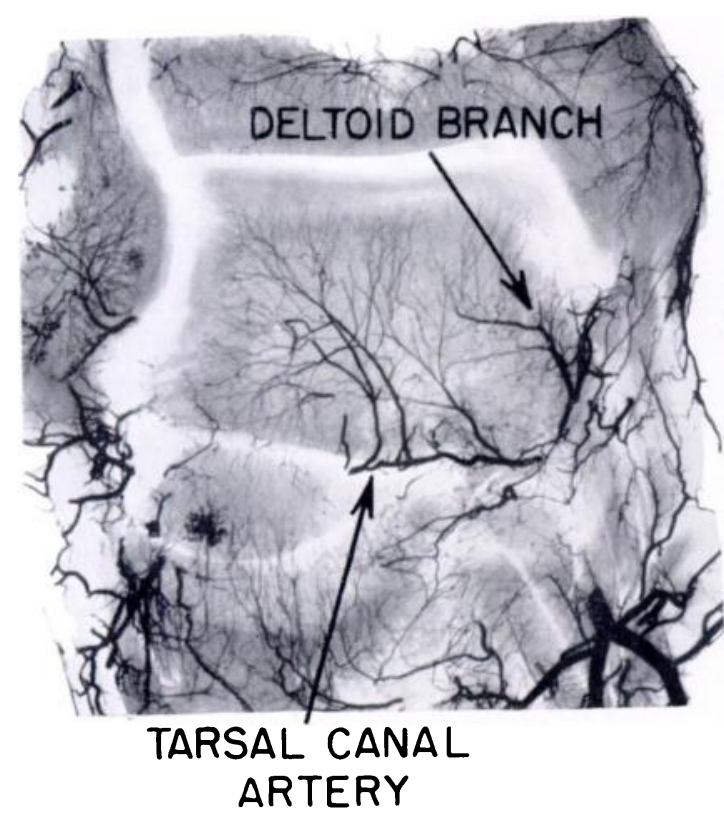

Fig. 6

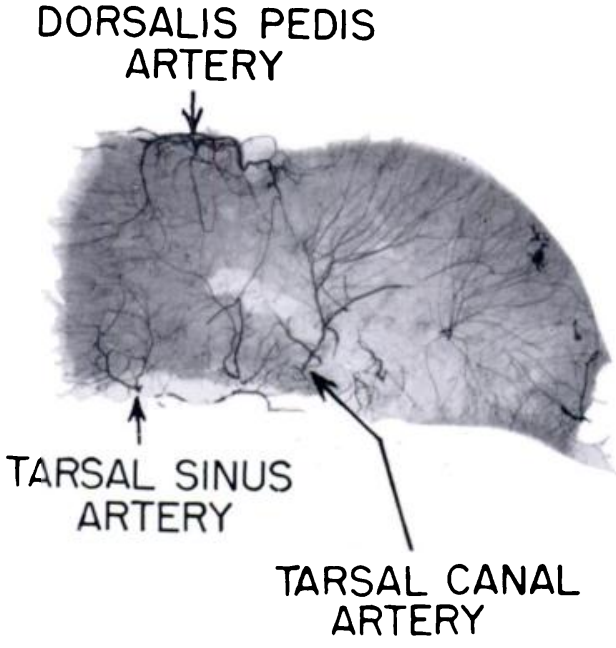

FiG. 7

Figure 6-A coronal section through the middle third of the talus. Figure 7-A sagittal section through the middle third of the talus. Anastomoses can be seen between the dorsalis pedis artery branches and the artery of the tarsal canal branches.

The arteries that enter the superior aspect of the neck usually send one or two branches into the middle of the anterior superior aspect of the trochlea (B in Fig. 1 and Fig. 3). 
There are several small branches from the posterior periosteal network which entered the posterior tubercle (B and C in Fig. 1). These supply only a small area limited to the posterior tubercle region.

Variations-In two specimens the deltoid branches were very large and supplied one-half of the body. In ten specimens the lateral branches from the tarsal sinus anastomotic network branched superiorly and supplied the lateral quarter of the body. In two tali the superior neck vessels were of large calibre and supplied approximately a third of the body although, in seven specimens, these vessels were not seen to penetrate the body at all.

Anastomoses-There were extensive arterial anastomoses around the ankle between the medial and lateral malleolar extraosseous networks. Injection of any one of the three larger arteries just above the ankle results in very rapid filling of the other two. There is a continuous arterial anastomosis in the periosteal network from the medial aspect of the posterior tubercle along the medial surface of the body to the superior aspect of the neck and then into the tarsal sinus (Fig. 4). The periosteal network ended on the lateral side of the body at the anterior edge of the articular surface for the lateral malleolus. The largest anastomotic arteries in periosteal network are between the deltoid branch of the artery of the tarsal canal and arteries on the superior aspect of the neck. As previously noted, the artery of the tarsal canal anastomoses with the artery of the tarsal sinus in the tarsal canal.

In the study of these sections, definite anastomoses were seen within the bone in eighteen of the thirty tali. The most common anastomosis was between the arteries entering the superior neck and those from the artery of the tarsal canal (Fig. 7). Eight were identified. Four had anastomosis between the inferior and superior vessels in the head (Fig. 5) and four had anastomosis between the artery of the tarsal sinus and the artery of the tarsal canal within the body. One anastomosis was between the posterior tubercle branches and the artery of the tarsal canal and one between the deltoid branches and the artery of the tarsal canal.

This probably represents only a small number of the potential arterial anastomoses because small arteries in all specimens were not equally well filled, which must have obscured some anastomoses; further, not all anastomotic channels are open simultaneously during life nor is it known what changes occur in the anastomoses after death. Also, following small vessels from one section of the specimen to another was difficult and anastomoses were undoubtedly missed because of this.

\section{DISCUSSION}

The results of this study on the vasculature of the talus are in general agreement with those of Wildenauer (1950), except in the origin of the deltoid branch which supplies the medial aspect of the body. Wildenauer described the origin of this vessel as from the posterior tibial artery, whereas in this series it originated from the artery of the tarsal canal in twenty o'it of thirty specimens.

This investigation has also confirmed the work of Haliburton et al. and further delineates the areas of the talus supplied by the various arteries. Haliburton et al. found the largest vessels to the body of the talus traversed antero-inferiorly through the neck and that the posterior tubercle supply equalled that of the lateral branches to the body. In this series it was found that the largest vessels entered the body from the tarsal canal posterior to the neck and that the posterior tubercle branches were consistently small and relatively insignificant when compared with the other arteries.

This anatomical study showed correlation with some of the clinical problems peculiar to this bone. Most fractures of the neck of the talus do not cause avascular necrosis of the body. The vessels entering the medial surface of the body, the anterior lateral vessels and at least part of the vessels in the tarsal canal should remain intact. Coltart (1952) reported no avascular necrosis with simple fracture of the neck. Mindell, Cisek, Kartalian and Dziob (1963) reported an incidence of 28 per cent of avascular necrosis of the body with simple fracture of the neck. 
The incidence of necrosis rises sharply with dislocation and fracture-dislocation of the body, which is what would be expected if the body loses all or most of its soft-tissue attachments. Avascular necrosis of the body of the talus after simple fracture of the neck must imply unrecognised soft-tissue damage around the talus with arterial occlusion from soft-tissue swelling or arterial rupture from partial dislocation.

Triple arthrodesis also interferes with the blood supply to the talus. The commonly employed surgical approach through the tarsal sinus obliterates the artery of the tarsal sinus and wide facet removal will likely damage the artery of the tarsal canal. A Lambrinudi triple arthrodesis removes all the vessels which enter the inferior surface, though the deltoid and superior neck vessels are unaffected.

In spite of the loss of major vessels, avascular necrosis of the talus is unusual. This tends to support the view that arterial anastomoses within the talus are numerous.

\section{SUMMARY}

1. The intraosseous and extraosseous circulation of the talus was examined in thirty necropsy specimens.

2. The blood supply to the talus is quite diffuse and arises from the three major arteries of the lower leg.

3. The common patterns of circulation, as well as the variations, have been documented.

\section{REFERENCES}

Ada(HI, B. (1928): Das Arteriensystem der Japaner. Vol. II, p. 247. Tokyo: Kenkyusha.

Coltart, W. D. (1952) “Aviator's Astragalus”. Journal of Bone and Joint Surgery, 34-B, 545.

Depreux, M. R., and Hollingshausen (1963): Vascularisation artérielle de l'astragale. Lille-chirurgical, $18,188$.

Haliburton, R. A., Sullivan, C. R., Kelly, P. J., and Peterson, L. F. A. (1958): The Extra-osseous and Intra-osseous Blood Supply of the Talus. Journal of Bone and Joint Surgery, 40-A, 1115.

Huber, J. F. (1941): The Arterial Network Supplying the Dorsum of the Foot. Anatomical Record, 80, 373. Kleiger, B. (1948): Fractures of the Talus. Journal of Bone and Joint Surgery, 30-A, 735.

Lauro, A., and Purpura, F. (1956): La trabecolatura ossea e l'irrorazione sanguigna nell'astragalo e nel calcagno. Minerva chirurgica, 11, 663.

LeXer, E., KUlıgA, P., and TürK, W. (1904): Untersuchungen über Knochenarterien mittelst Röntgenaufnahmen injizierter Knochen und ihre Bedeutung für einzelne pathologische Vorgänge am Knochensysteme, p. 16. Berlin: August Hirschwald.

McKeever, F. M. (1943): Fracture of the Neck of the Astragalus. Archives of Surgery, 46, 720.

Mindell, E. R., Cisek, E. E., Kartalian, G., and Dziob, J. M. (1963): Late Results of Injuries to the Talus. Journal of Bone and Joint Surgery, 45-A, 221.

Montis, S., and Ridola, C. (1959): Vascolarizzazione Dell'Astragalo. Quaderni di Anatomia Pratica, $15,574$. Phemister, D. B. (1940): Changes in Bones and Joints Resulting from Interruption of Circulation; I. General Considerations and Changes Resulting from Injuries. Archives of Surgery, 41, 436.

SNeED, W. L. (1925): The Astragalus. A Case of Dislocation, Excision and Replacement. An Attempt to Demonstrate the Circulation on this Bone. Journal of Bone and Joint Surgery, 7, 384.

Trueta, J., and Harrison, M. H. M. (1953): The Normal Vascular Anatomy of the Femoral Head in Adult Man. Journal of Bone and Joint Surgery, 35-B, 442.

WATSON-JONeS, R. (1940): Fractures and Other Bone and Joint Injuries, p. 77. Edinburgh: E. \& S. Livingstone. Wildenauer, E. (1950): Die Blutversorgung des Talus. Zeitschrift für Anatomie und Entwicklungsgeschichte, $115,32$. 